\title{
Threshold Secure B2B Model
}

\author{
C. Phani Ramesh ${ }^{1}$, Prof. M. Padmavathamma ${ }^{2}$ \\ 1,2(Department of Computer Science, Sri Venkateswara University, Tirupati, A.P., India) \\ ('phaniramesh@gmail.com, ${ }^{2}$ prof.padma@yahoo.com)
}

\begin{abstract}
E-Commerce plays a significant role in the area of Distributed Networks and Data Mining. Businessto-Business (B2B) electronic commerce is now universally recognized as the ecommerce segment with the largest potential. The growth of the Internet and commercial Web-based applications is offering ever-increasing operational cost savings to large enterprises, extending trading communities and lowering the financial barriers to ecommerce participation. In this paper, we cleanly focus on Business to Business framework for selecting the best Distributor in a secure manner among the available Distributors in B2B Scenario.
\end{abstract}

Keywords: E-Commerce, Business to Business, Security, Development Framework, Decision making.

\section{Introduction}

Information and knowledge have become key strategic resources, upon which organizations across all industries make their decisions. Trends that have made information systems of strategic importance include globalization and competitive pressures for increased quality with lower costs. In the global business environment, businesses should see the enhanced role of electronic business as particularly increasing the importance of information systems. The Internet with its open environment, and other networks have made it possible for the organization to access and exchange enormous amounts of electronic information both inside in the organization and around the world with minimal time resulting in lower communication and coordination costs [1].

In the emerging Global Economy, E-commerce and E-business have increasingly become a necessary component of business strategy and a strong catalyst for economic development. The security of Internet electronic transactions is one of the key factors needed for further E-business development. Online Commerce processes demonstrate particularly to security problems.

In this paper, the proposed framework focuses on the secure classification, verification and development of decision tree for selecting the Best Distributor among the available Distributors by the Manufacturer in B2B Scenario.

\section{Related Work}

With the advance of Internet technology, e-businesses are striving to reach an unprecedented large population and start to take on new forms. An e-business can be built on top of a wide range of e-business models.

E-commerce (electronic commerce) can be defined as the summation of all commercial activities being performed in, via or by facilitation of virtual space, i.e. Internet or the World Wide Web. All commercial activities running inside the electronic-business sphere can be regarded as E-commerce. E-Business commonly represents all types of commercial activities which are based on the Internet such as retail shopping centers, banking, stocks and bonds trading, auctions, real estate transactions, plane ticket booking, movie rentals and anything that could be operated in real world. Not only are some commercial transactions done directly in the electronic sphere, but personal services or other real world business are also represented online on the Internet. This way, the E-Business is growing as an independent, sophisticated and enormous operational section of the world economy[2].

As online E-commerce continues to grow in all areas, customer expectations particularly in B2B ecommerce are changing rapidly.

\subsection{BUSINESS-TO-BUSINESS (B2B):}

It describes commerce transactions between businesses, such as between a manufacturer and Distributor or between a wholesaler and a Distributor. B2B transactions primarily target companies and other wholesale distributors. A business-to-business (B2B) wholesale distributor refers to a business that moves products through the supply chain by purchasing from manufacturers and selling to retailers. The B2B e-Marketplace can significantly improve the way companies deal with their customers and distributors. [3] 


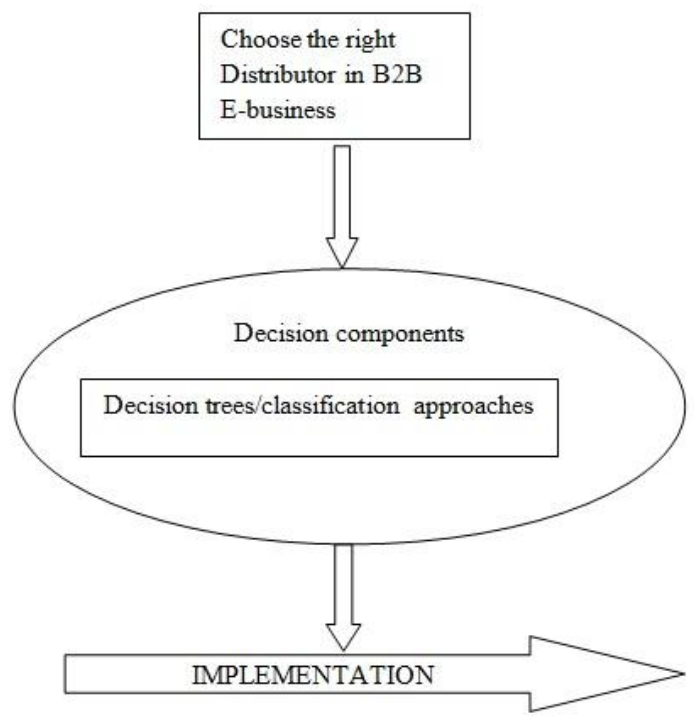

\subsection{DECISION SYSTEM:}

We select classification approaches based on their knowledge discovery capability in general and knowledge interpretability in particular. Rules usually consist of several if patterns and one or more then patterns. They are known as straightforward and easy to understand and verify, and thereby can be easily applied by Manufacturers. They can represent associations and causal-effect relationships between different variables or factors [4]. Knowledge in form of decision tree can reveal patterns in distributors' perception of Internet retailing services. Therefore, we target at decision tree in this study.

This paper takes a step toward discovering knowledge for predicting distributors in b2bby using classification approaches. We select classification approaches based on their knowledge discovery capability in general and knowledge interpretability in particular.

\section{Decision Trees}

Decision trees [5] classify instances by sorting them down the tree from the root to some leaf node, which provides the classification of the instance. Each node in the tree specifies a test of some attribute of the instance and each branch descending from that node corresponds to one of the possible values for this attribute.

The reasons for decision learning tree algorithms to be attractive are: -

1. They generalize in a better way for unobserved instances, once examined the Attribute value pair in the training data.

2. They are efficient in computation as it is proportional to the number of training Instances observed.

3. The tree interpretation gives a good understanding of how to classify instances based on attributes arranged on the basis of information they provide and makes the classification process self-evident.

ID3 is a decision tree algorithm which constructs decision tree by employing a top-down, greedy search through the given sets of training data to test each attribute at every node [6]. It uses statistical property call information gain to select which attribute to test at each node in the tree. Information gain measures how well a given attribute separates the training examples according to their target classification.

\section{ENTROPY}

It is a measure in the information theory, which characterizes the impurity of an arbitrary collection of examples. If the target attribute takes on $\mathrm{c}$ different values, then the entropy $\mathrm{S}$ relative to this $\mathrm{c}$-wise classification is defined as

$\operatorname{Entropy}(\mathrm{S})=\sum_{i=1}^{c}-p_{i} \log _{2} p_{i}$

where pi is the proportion/probability of $\mathrm{S}$ belonging to class i. Logarithm is base 2 because entropy is a measure of the expected encoding length measured in bits.

\section{INFORMATION GAIN}

It measures the expected reduction in entropy by partitioning the examples according to this attribute. The information gain, Gain(S, A) of an attribute A, relative to the collection of examples $\mathrm{S}$, is defined as 
$\operatorname{Gain}(\mathrm{S}, \mathrm{A})=$ Entropy $(\mathrm{S})-\sum_{\text {vevalues }(A)} \frac{s v}{s} \operatorname{Entropy}\left(S_{v}\right)$

where Values ( A) is the set of all possible values for attribute A, and Sv is the subset of S for which the attribute A has value $\mathrm{v}$. We can use this measure to rank attributes and build the decision tree where at each node is located the attribute with the highest information gain among the attributes not yet considered in the path from the root.

\section{Proposed Secure B2b Model}

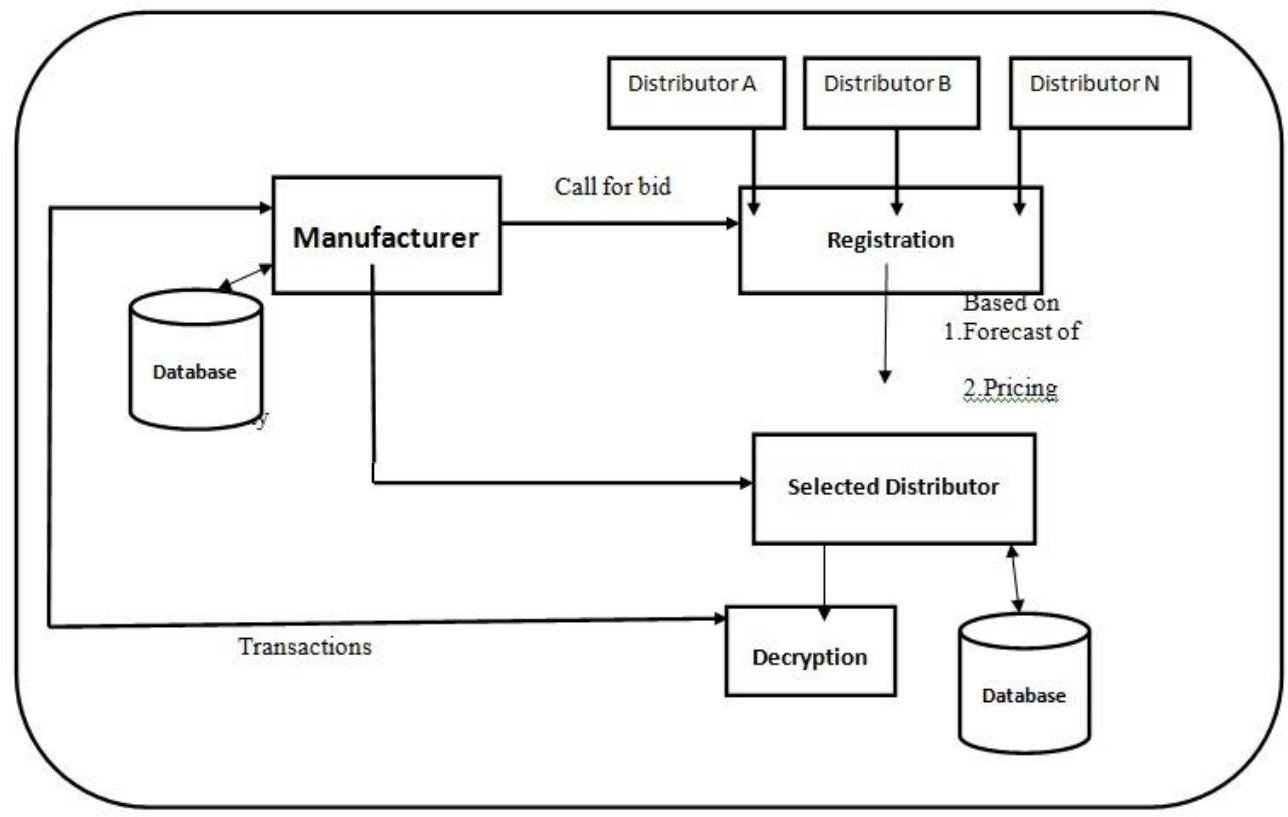

From the above System Model, the Manufacturer is responsible for the effective promotion of Business Products. This requires a thorough knowledge of the products being promoted, as well as the ability to solicit orders from outside Distributors, the manufacturer calls for bid from the Distributors. There are $\mathrm{n}$ distributors $(=\mathrm{d} 1, \mathrm{~d} 2, \mathrm{~d} 3, \ldots . . \mathrm{dn})$ and Manufacturer $\left(\mathrm{M}_{\mathrm{B}}\right)$. The role of Manufacturer is to organize each auction, run and announces the bid result based on the attributes Forecast of Purchase (FP), Marketing Knowledge (Mk), Payment History (PH), Manufacturer Relationships (MR) and Advertising Support (As) using decision trees. The channels between Manufacturer and Distributor are to secure and reliable.

The above model consists of the following phases

1) Registration phase: The Manufacturer calls for bid from the Distributors. The Distributors will make registrations here.

2) Announce phase: The manufacturer runs the bid by using the number of attributes and announces the selected distributor among the registered distributors.

THE SYMBOLIC ATTRIBUTE DESCRIPTION:

\begin{tabular}{|l|l|}
\hline \multicolumn{1}{|c|}{ Attributes } & \multicolumn{1}{c|}{ Possible Values } \\
\hline Forecast of Purchase (FP) & High, Low, Medium \\
\hline Marketing Knowledge (MK) & Good, Minimal, Low \\
\hline Payment History (PH) & Paid, Not Paid, Moderate \\
\hline Manufacturer Relationship (MR) & Maintains, Not Maintains \\
\hline Advertising Support (AS) & Excellent, Satisfactory, Poor \\
\hline Decision & Yes, No \\
\hline
\end{tabular}


Threshold Secure B2B Model

THE LEARNING SET TO SELECT THE BEST DISTRIBUTOR EXAMPLE:

\begin{tabular}{|c|l|l|l|l|l|l|}
\hline Distributors & $\begin{array}{l}\text { Market } \\
\text { Knowledge } \\
\text { (Mk) }\end{array}$ & $\begin{array}{l}\text { Payment } \\
\text { History } \\
\text { (PH) }\end{array}$ & $\begin{array}{l}\text { Forecast of } \\
\text { Purchases } \\
\text { (FP) }\end{array}$ & $\begin{array}{l}\text { Manufacturer } \\
\text { Relationships } \\
\text { (MR) }\end{array}$ & $\begin{array}{l}\text { Advertising } \\
\text { Support(AS) })\end{array}$ & Decision \\
\hline 1 & Good & Paid & High & Maintains & Excellent & Yes \\
\hline 2 & Low & Not paid & Low & Not Maintains & Satisfactory & No \\
\hline 3 & Minimal & Moderate & Low & Not Maintains & Poor & No \\
\hline 4 & Low & Paid & High & Maintains & Excellent & No \\
\hline & Good & Paid & Medium & Not Maintains & Satisfactory & Yes \\
\hline 6 & Low & Not Paid & Medium & Maintains & Excellent & Yes \\
\hline 7 & Good & Paid & Low & Not Maintains & Satisfactory & No \\
\hline
\end{tabular}

\section{DISTRIBUTOR DECISION TREE}

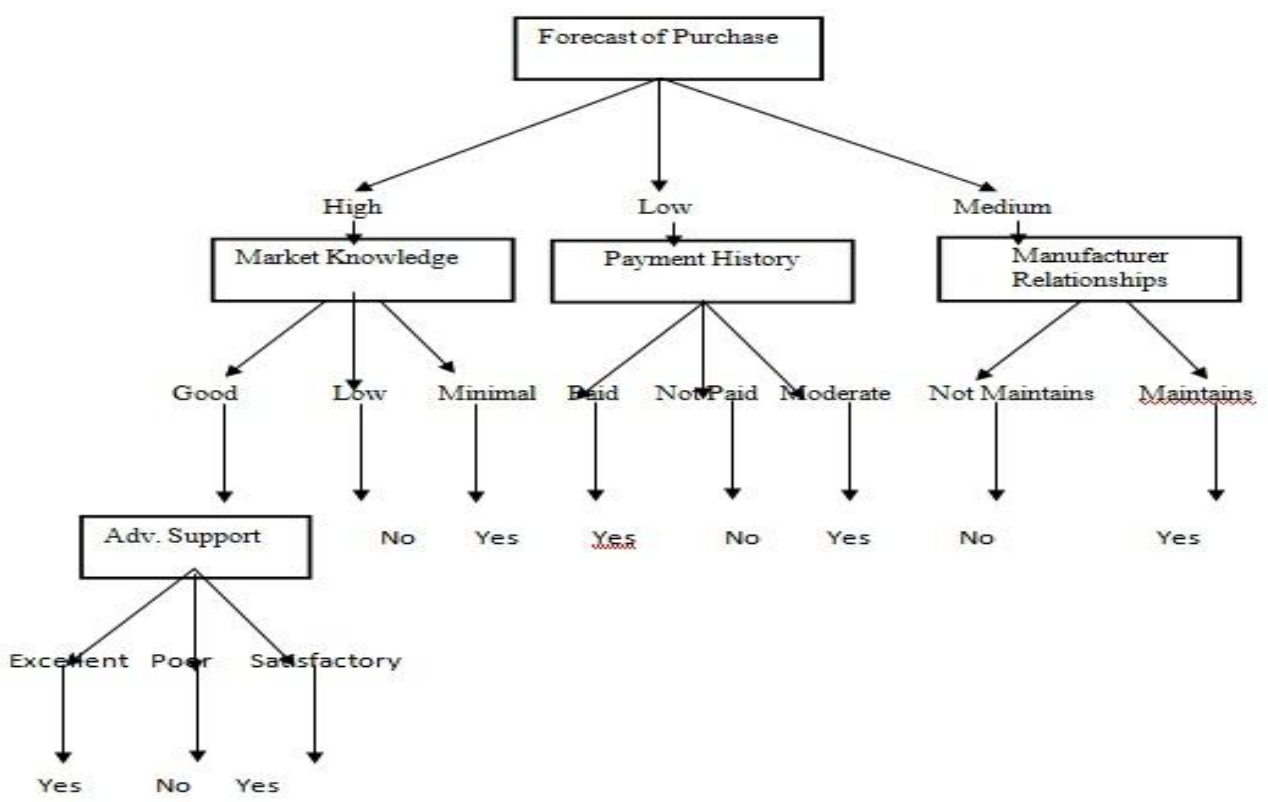

\section{Conclusion}

Classification techniques have been widely applied to facilitating decision making in an e-business environment. In this paper, we select the classification approach decision tree for making good decision for selecting the best distributor among the available distributors in a secure manner in B2B Scenario.

\section{References}

[1] Moses Niwe, Standards-based B2B e-Commerce Adoption, Advances in System Modeling and ICT Applications.

[2] Sabir Said, E-commerce Solution - Creating a shopping cart using Open sources, bachelors diss.,Turku University of Applied Sciences", 2010

[3] Ethan Lieber, Chad Syverson, Online vs. Offline Competition (Oxford Handbook of Digital Economy, 2011).

[4] Lina Zhou, Wei-yu Kevin Chiang, Dongsong Zhang, Discovering Rules for Predicting Customers Attitude toward Internet Retailers, Journal of Electronic Commerce Research, VOL. 5, NO.4, 2004

[5] Zhiguand Wang, Review of basic Machine learning approaches

[6] Saher Esmeir, Shaul Markovitch, Anytime Learning of Decision Trees, Journal of Machine Learning Research 8 (2007) $891-933$ 